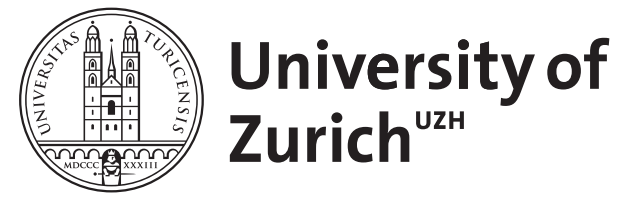

\title{
Secukinumab for Acrodermatitis Continua of Hallopeau
}

Muggli, David ; Maul, Julia-Tatjana ; Anzengruber, Florian ; Fopp, Myriam Wyss ; Navarini, Alexander A

DOI: https://doi.org/10.1001/jamadermatol.2016.5059

Posted at the Zurich Open Repository and Archive, University of Zurich ZORA URL: https://doi.org/10.5167/uzh-138150

Journal Article

Published Version

Originally published at:

Muggli, David; Maul, Julia-Tatjana; Anzengruber, Florian; Fopp, Myriam Wyss; Navarini, Alexander A (2017). Secukinumab for Acrodermatitis Continua of Hallopeau. JAMA Dermatology, 153(4):336-337. DOI: https://doi.org/10.1001/jamadermatol.2016.5059 


\section{Letters}

\section{OBSERVATION}

\section{Secukinumab for Acrodermatitis Continua of Hallopeau}

Acrodermatitis continua of Hallopeau (ACH) was first described in 1890 by Henri Hallopeau. ${ }^{1}$ He had observed a single patient with an undulating but recalcitrant pustular destruction of the fingertips and toes. ${ }^{1}$ Nowadays, this condition is considered a subtype of localized pustular psoriasis.

There are no treatment guidelines available for $\mathrm{ACH}$, and the condition is refractory to many conventional topical and systemic treatments. ${ }^{2}$ Several patients have been treated with biologic agents with variable success. Targeted treatment strategies based on pathogenic cytokine expression patterns have not been evaluated for ACH.

Report of a Case | A 87-year-old retired electrical engineer had recurrent episodes of redness, swelling, and purulent discharge in the area of the left hallux with progressive degeneration of the toenail for 2 years. Other digits were not in- volved. Antibiotic treatment had been ineffective. The affected toe showed onychodystrophy, and the erythematous perionychium was beset with pustules (Figure 1A). All other toes and fingers were normal. Findings of mycologic and bacterial swabs were negative. No signs of systemic inflammation were present.

A medial longitudinal biopsy of the nail bed of the left hallux revealed numerous neutrophils within the stratum corneum, psoriasiform dermatitis with epidermal hyperplasia, a lacking granular layer, and compact hyperkeratosis and parakeratosis. These findings are compatible with the diagnosis of acrodermatitis continua of Hallopeau (ACH). The patient, a nonsmoker, did not have plaque-type psoriasis but had a family history of this condition. After ineffective treatment with topical steroids, UV-B phototherapy, and acitretin for 9 months, the ACH flared to involve all fingers (Figure 1B). The Nail Psoriasis Severity Index score reached the maximum score of 80 points. On the pain visual analog scale, the patient reported the maximum score, 10 of 10 .

\section{Figure 1. Clinical Findings}

A At presentation

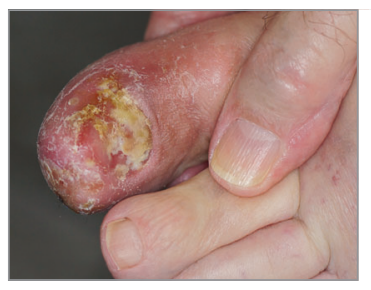

B Nine months after initial presentation

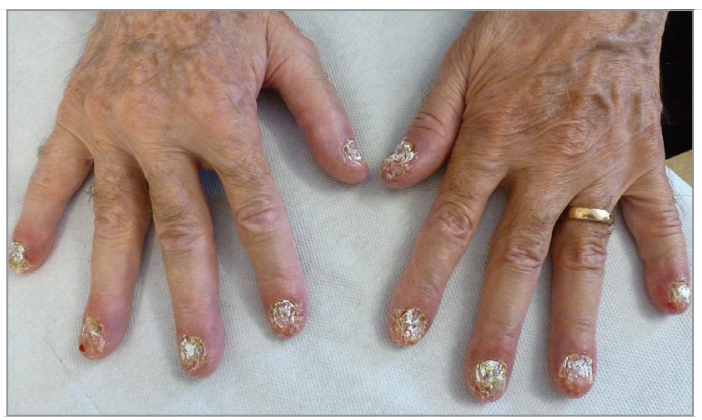

C After treatment

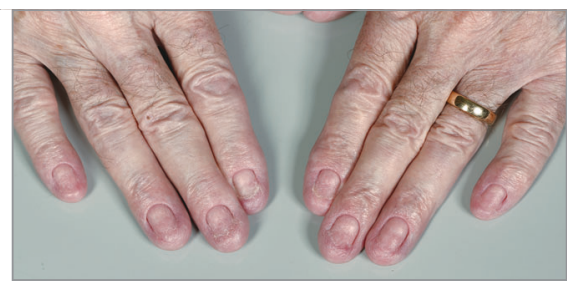

A, Patient's left big toe on initial presentation. B, Involvement of the fingers 9 months later and shortly before initiation of treatment with secukinumab. C, Nine weeks after treatment with secukinumab.

\section{Figure 2. Histopathologic Findings}

A Normal skin

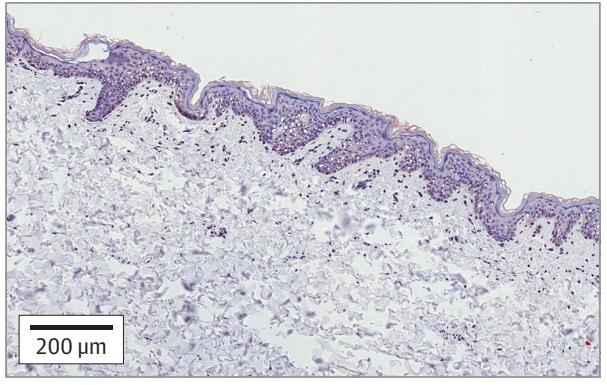

B Affected skin

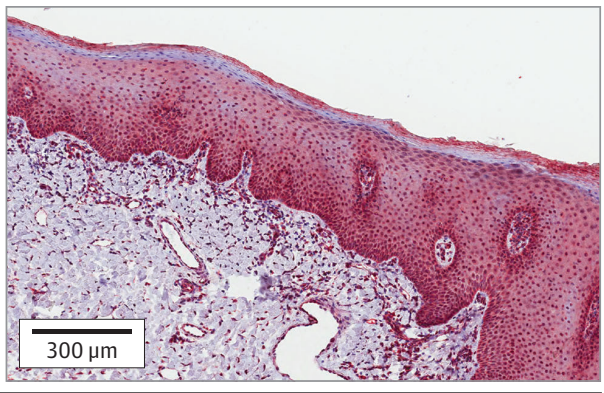

A, Normal (uninvolved) non-acral skin sample (interleukin [IL]-17 stain, original magnification $\times 400$ ). $\mathrm{B}$, Acrodermatitis continua of Hallopeau (involved) skin sample from the left big toe (IL-17 stain, original magnification $\times 400$ ). 
In generalized pustular psoriasis (GPP), interleukin (IL)-17 mRNA levels are elevated. ${ }^{3}$ Even though they are distinct clinical entities, ACH and GPP are closely related on the genetic level. ${ }^{4}$ Therefore, IL-17 levels in affected skin were evaluated in search of a therapeutic target, and strong IL-17A expression was found (Figure 2). Therapeutic inhibition of this cytokine with the monoclonal antibody secukinumab was evaluated in the registered dose for plaque-type psoriasis (300 mg subcutaneously in weeks 0 , $1,2,3$, and 4 and every 4 weeks thereafter), and based on the results of this evaluation, the patient was treated exclusively with secukinumab for 6 weeks. At 6 weeks, complete resolution of inflammation in all affected fingers was observed, and the fingernails had begun to regrow (Figure 1C). The patient was satisfied with the therapy, reporting a decrease in visual analog scale pain score from 10 to 2 and an improvement of the Dermatology Life Quality Index from 7 to 4 points. No adverse effects were reported during the course of the therapy.

Discussion | The cytokine IL-17 plays a key role in chronic inflammation and psoriasis pathogenesis. It acts directly on keratinocytes and induces epidermal hyperplasia, acanthosis, and hyperparakeratosis and leads to recruitment and accumulation of neutrophils, T cells, and dendritic cells. Furthermore, IL-17 is known to enhance local neutrophil activity under inflammatory conditions. An open-label trial of the IL-17 inhibitor ixekizumab showed efficacy in moderate to severe GPP, ${ }^{5}$ and very recently, a case report demonstrated activity of secukinumab in GPP. ${ }^{6}$ Therefore, treatment trials with inhibition of IL-17 are warranted in this orphan disease. In addition, at least theoretically, some degree of individualization of targeted therapies could be achievable by detection of pathogenic cytokine expression, such as performed here by immunohistochemical analysis for IL-17A in affected tissue. This should be addressed in future randomized trials.
David Muggli, MD

Julia-Tatjana Maul, MD

Florian Anzengruber, MD

Myriam Wyss Fopp, MD

Alexander A. Navarini, MD, PhD

Author Affiliations: Department of Dermatology, University Hospital Zurich, Zurich, Switzerland (Muggli, Maul, Anzengruber, Navarini); Center for Laser and Aesthetic Medicine, Meilen, Switzerland (Fopp).

Corresponding Author: Alexander A. Navarini, MD, PhD, Department of Dermatology, University Hospital Zurich, Gloriastrasse 31, CH-8091 Zurich, Switzerland (alexander.navarini@usz.ch).

Published Online: January 25, 2017. doi:10.1001/jamadermatol.2016.5059

Conflict of Interest Disclosures: None reported.

Funding/Support: This study was supported by the Department of Dermatology, University Hospital of Zurich.

Role of the Funder/Sponsor: The funder had no role in the design and conduct of the study; collection, management, analysis, and interpretation of the data; preparation, review, or approval of the manuscript; and decision to submit the manuscript for publication.

Additional Contributions: We thank the patient for granting permission to publish this information.

1. Hallopeau MH. Sur une asphyxie locale des extremitiés avec polydactylite suppurative chronique et poussées ephemères de dermatite pustuleuse disseminée et symetrique. Soc Fr Dermatol Syph Bull. 1890;(1):39-45.

2. Puig L, Barco D, Vilarrasa $E$, Alomar A. Treatment of acrodermatitis continua of Hallopeau with TNF-blocking agents: case report and review. Dermatology. 2010;220(2):154-158

3. Lee E, Zarei M, LaSenna C, Villada G, Romanelli P. Psoriasis targeted therapy: characterization of interleukin 17A expression in subtypes of psoriasis. $J$ Drugs Dermatol. 2015;14(10):1133-1136.

4. Abbas $\mathrm{O}$, Itani $\mathrm{S}$, Ghosn $\mathrm{S}$, et al. Acrodermatitis continua of Hallopeau is a clinical phenotype of DITRA: evidence that it is a variant of pustular psoriasis. Dermatology. 2013;226(1):28-31.

5. Saeki $H$, Nakagawa $H$, Ishii T, et al. Efficacy and safety of open-label ixekizumab treatment in Japanese patients with moderate-to-severe plaque psoriasis, erythrodermic psoriasis and generalized pustular psoriasis. J Eur Acad Dermatol Venereol. 2015;29(6):1148-1155.

6. Böhner A, Roenneberg S, Eyerich K, Eberlein B, Biedermann T. Acute generalized pustular psoriasis treated with the il-17a antibody secukinumab. JAMA Dermatol. 2016:152(4):482-484 